\title{
Influenza and obesity: its odd relationship and the lessons for COVID-19 pandemic
}

\author{
Livio Luzi $^{1,2} \cdot$ Maria Grazia Radaelli ${ }^{1}$
}

Received: 17 March 2020 / Accepted: 18 March 2020 / Published online: 5 April 2020

(c) Springer-Verlag Italia S.r.l., part of Springer Nature 2020

\begin{abstract}
Aims Analyze the relationship between obesity and influenza.

Methods Basal hormone milieu, defective response of both innate and adaptive immune system and sedentariness are major determinants in the severity of influenza viral infection in obese patients. Being overweight not only increases the risk of infection and of complications for the single obese person, but a large prevalence of obese individuals within the population might increase the chance of appearance of more virulent viral strain, prolongs the virus shedding throughout the total population and eventually might increase overall mortality rate of an influenza pandemic.

Results Waiting for the development of a vaccination against COVID-19, isolation of positive cases and social distancing are the primary interventions. Nonetheless, evidence from previous influenza pandemics suggests the following interventions aimed at improving immune response: (1) lose weight with a mild caloric restriction; (2) include AMPK activators and PPAR gamma activators in the drug treatment for obesity associated with diabetes; and (3) practice mild-to-moderate physical exercise.

Conclusions Due to prolonged viral shedding, quarantine in obese subjects should likely be longer than normal weight individuals.
\end{abstract}

Keywords Obese subjects $\cdot$ COVID-19 $\cdot$ Influenza $\cdot$ Immune-modulation

\section{Introduction}

COVID-19 epidemic is caused by an influenza-like virus strain (SARS-CoV-2). Since the "Spanish" influenza pandemic of 1918, it is known that malnutrition (both underand over-nutrition) is linked to a worse prognosis of the viral infection [1]. The 1957-1960 "Asian" and the 1968 "Hong Kong" influenzas confirmed that obesity and diabetes lead to a higher mortality as well as a more prolonged duration of illness even if the subjects were without other chronic conditions that increase the risk of influenza-related complications [2, 3]. During the 2009 Influenza A virus (IAV) H1N1

Managed By Antonio Secchi.

Livio Luzi

livio.luzi@multimedica.it; livio.luzi@unimi.it

1 Department of Endocrinology, Nutrition and Metabolic Diseases, IRCCS MultiMedica, Milan, Italy

2 Department of Biomedical Sciences and Health, Università degli Studi di Milano, Milan, Italy pandemic, obesity was also linked to increased risk of severe disease and a risk factor for hospitalization and death [4].

In industrialized countries, about $50 \%$ of the population is overweight or obese, with prevalence increasing annually. In the USA, the last NHANES report indicated that 34\% Americans are obese according to WHO criteria [5]. One model from the Center for Disease Control and prevention (CDC) suggested that between 160 million and 210 million Americans could contract the disease over a 12-month period. Based on mortality data and current hospital capacity, the number of deaths under the CDC's scenarios ranged from 200,000 to as many as 1.7 million [6].

Herein, we will present some considerations and evidence concerning the impact of the high prevalence of obesity in the diffusion and perpetuation of the epidemics of COVID-19. 


\section{Why do obese patients present a worse clinical picture than lean subjects?}

Obese and obese-diabetic subjects have an alteration at different steps of the innate and adaptive immune response, characterized by a state of chronic and low-grade inflammation [7]. This pathway contributes to systemic metabolic dysfunction that is associated with obesity-linked disorders.

Firstly, obese subjects have chronically higher leptin (a pro-inflammatory adipokine) and lower adiponectin (an anti-inflammatory adipokine) concentrations. This unfavorable hormone milieu also leads to a dysregulation of the immune response and can contribute to the pathogenesis of obesity-linked complications [8]. In the basal state, obese patients have a higher concentration of several pro-inflammatory cytokines like alpha-TNF, MCP-1 and IL-6, mainly produced by visceral and subcutaneous adipose tissue leading to a defect in the innate immunity [9]. When an antigen is presented, the obesity-related chronic inflammation causes a reduced macrophage activation and a blunted pro-inflammatory cytokine production upon macrophage stimulation [10]. The reduced macrophage activation after an antigen is presented explaining the poor vaccination success of obese subjects [11]; this unique obesogenic microenvironment explains the emergence of antiviral-resistant and vaccine escape variants in the obese population $[11,12]$. Also $\mathrm{B}$ and $\mathrm{T}$ cell responses are impaired in obese and obese-diabetic patients, and this causes an increased susceptibility and a delay of resolution of the viral infection.

Zhang et al. proposed that leptin resistance was a cofactor for the 2009 A (H1N1) pandemic influenza, leptin being a major regulator of $\mathrm{B}$ cell maturation, development and function [13]. Similarly, obese patients may present with numerical and functional alterations of lymphocytes leading to impaired memory $\mathrm{T}$ cell responses and vaccine efficacy [14]. Obesity inhibits both virus-specific CD8 + T cell responses and antibody responses to the seasonal influenza vaccine; again a suboptimal macrophage functionality and maturation characteristic of the obese host may contribute to the poor vaccine response [15].

A dysregulated pro-inflammatory response contributes to the severe lung lesions observed in victims during the influenza pandemic. A robust $\mathrm{CD} 8+\mathrm{T}$ cell response plays an important role in protection against novel influenza virus strains and subtypes.

Several studies on IAV infection in OB mice show increased disease severity, increased secondary bacterial infections and a reduced vaccine efficacy [16]. In fact, in obese mice there is a delayed and blunted immune response, probably due to a reduced interferon (INF), adaptive cellular and antibody-mediated responses. Recently, Honce et al. found that serial passaging of a human H1N1 influenza virus through diet-induced models of obesity mice (DIO) or genetically obese mice (OB) results in a more virulent disease with an increased virulence and morbidity; in the mouse model, deep-sequencing viruses revealed several mutations in obese host-passaged viruses and an impairment of the interferon response is permissive for the generation of more virulent IAV populations. These findings are not limited by viral subtype and are also found in human studies. Diminished INF response and an increased influenza virus replication were found in normal bronchial epithelial (NHBE) cells derived from obese subjects [16-18].

Another important problem among obese subjects is physical inactivity. Sedentariness or reduced physical activity is characteristic of obese patients with respect to lean subjects. Reduced physical activity per se [19] or mediated by insulin resistance [20] impairs immune response against microbial agents at several steps of the immune response, including macrophage activation and inhibition of proinflammatory cytokines.

Both physical activity and exercise are positively associated with favorable outcomes in metabolic (diabetes, obese and metabolic syndrome) and immunological (immune activation levels, vaccination efficacy and immune senescence) health; physical activity interventions have demonstrated the potential to reduce the risk of complications by modulating inflammation, augmenting the immune system and improving vaccination outcomes in the elderly [21].

It is also important to consider as a potential cause of the worse clinical picture of obese individuals the dynamic of pulmonary ventilation in these subjects, with reduced diaphragmatic excursions and a relative increase in anatomical death space.

\section{Why are obese patients potentially more contagious than lean subjects?}

Three factors make obese subjects more contagious than leans: First, obese subjects with influenza shed the virus for a longer period of time (up to $104 \%$ longer) than lean subjects, potentially increasing the chance to spread the virus to others [22]. Secondly, the obese microenvironment favors the emergence of novel more virulent virus strains. This is due mainly to the reduced and delayed capacity to produce interferons by obese individuals and animals [17, 18]. The delay in producing interferon to contrast viral replication allows more viral RNA replication increasing the chances of the appearance of novel, more virulent viral strains [18]. Thirdly, body mass index correlates positively with infectious virus in exhaled breath [23]. This finding was 
especially significant for males, which leads to the hypothesis that the higher ventilation volumes or a differential chest conformation might explain this fact [23].

\section{Can a virus infection induce weight gain?}

The relationship between viral infections and obesity is more complex than a simple worsening of the influenza infectiveness and clinical picture and outcome in obese patients. Adenovirus 36 (Adv36) is the most studied infectious agent probably associated to obesity. Adenoviruses are DNA viruses causing mainly respiratory infections which were indicated both in animal models and in humans to be contributing factors for increased adiposity [24] (Fig. 1). Atkinson et al. showed in over 500 individuals and 28 twin pairs a correlation between BMI and Adv36 positivity, with $30 \%$ of positivity in obese vs $11 \%$ positivity in lean individuals [24]. The proposed mechanisms via which Adv36 infection may lead to obesity are several: First, the increase in chronic inflammation via rise in MCP-1 (macrophage chemoattractant protein I) which is activated by NF-kB [25]. Second, a reduction in leptin expression in the adipocytes leading to lipid accumulation by a decrease in lipolysis, a downregulation of the adipocyte genes involved in lipid oxidation and fatty acid synthesis [26]. Third, the increase in glucose uptake in the adipocytes leads to an energy surplus in the cells glucose-dependent [27]. Fourth, an up-regulation of adipogenesis is due to an improvement in adipocyte proliferation and differentiation [28]. Particularly, PPAR gamma genes and other RNA expressions factors related to adipocyte genesis increase.

The effects of influenza virus on infected mammalian cells generally result in a metabolic shift toward an increase in glycolytic rate and a decrease in ATP production [29]. The authors hypothesize that the onset of apoptosis causes this metabolic imbalance during the late stage of the influenza virus replication process [29]. This pattern resembles an insulin-resistant condition with a reduction in the mitochondrial pyruvate dehydrogenase complex (PDC) activity, usually involved in the rate of carbohydrate oxidation control. PDC is an important modulator of energy and metabolic homeostasis, and insulin resistance is associated with increased skeletal muscle pyruvate dehydrogenase kinase (PDK) that phosphorylates and inactivates PDC. Increased PDK4 expression in muscle has been observed in insulinresistant status, including obesity $[14,30]$. In mice infected by the H1N1 virus, there is a marked down-regulation of PDC activity and ATP level, with selective up-regulation of PDK4 in the skeletal muscles, heart, liver and lungs.

Influenza is characterized by cytokine storm and metabolic disorders. In this contest PDC, have an important role by catalyzing oxidative decarboxylation of pyruvate and linking glycolysis to the tricarboxylic acid cycle (TCA) and fatty acid synthesis. In mice, Yamane et al. [31] demonstrate that oral administration of dichloroacetate (DADA, a PDK4 inhibitor) restored PDC activity and ATP level, ameliorated metabolism disorders, suppressed cytokine storm, trypsin up-regulation and viral replication.

\section{What are the lessons to be learned from previous influenza virus infections in obesity?}

Incubation time for COVID-19 is considered 14 days, at most. Based on the concept of prolonged shedding of the virus in obese subjects [22], quarantine should be prolonged in obese with respect to lean subjects, presumably doubled. Until a specific vaccine is not available, the only actions that can be taken are isolation of positive patients and potentiation of positive immune modulation response. Diet and mainly physical exercise as immune modulatory action not only aimed at losing weight but also at quickly
Fig. 1 The figure schematizes the bidirectional relationship between influenza/influenzalike viral infections and obesity. Adenovirus 36 was linked to the development of obesity. Obese condition is characterized by higher viral charge in breath and prolonged virus shedding. Lifestyle interventions and drugs commonly used to treat diabetes associated with obesity, cause a positive immune-modulation

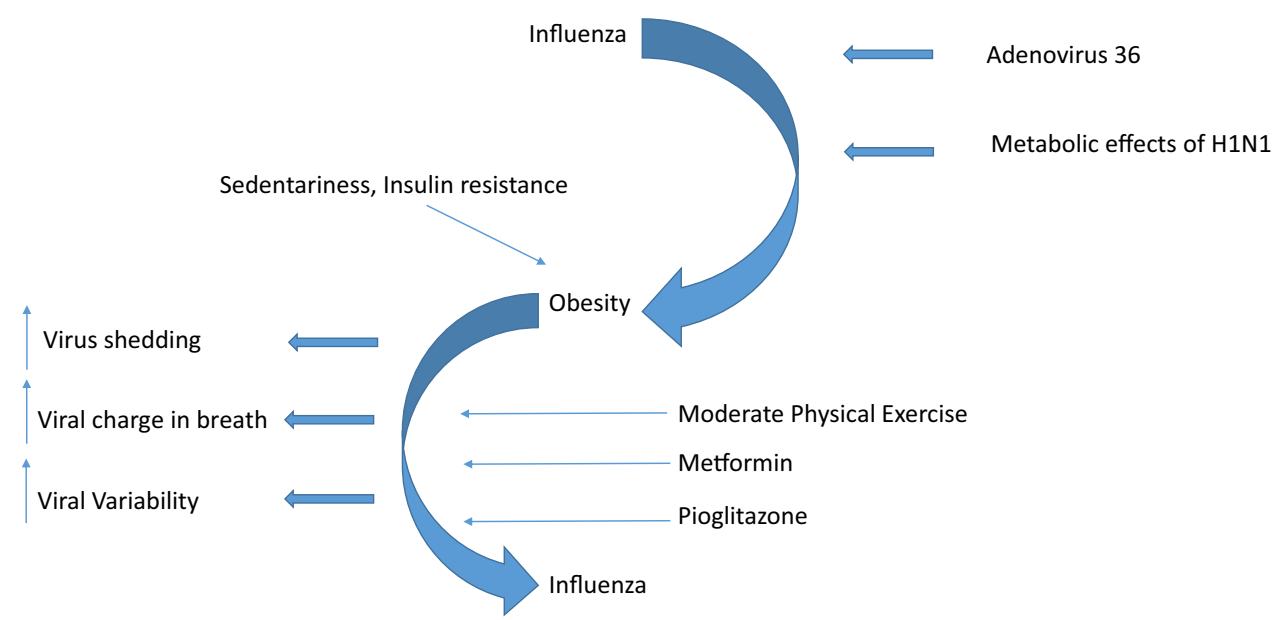


improving immune modulation; morbidity and mortality may, in fact, be reduced with regular exercise, even without substantial weight loss. Several studies suggest that regular physical exercise enhances levels of cytokines production mediated via TLR (toll-like receptor) signaling pathways during a microbial infection, improving host resistance to pathogen invasion [19]. An additional benefit of exercise is the enhancement of the antioxidant defense system and the oxidative stress reduction.

Warren et al. demonstrate that exercise improved host immunity to IAV infection in both obese and non-obese mice. Particularly, in obese mice, physical activity reversed the obesity-associated impairments in host-immune defense [32].

The results suggest that physical activity increased immune activation (BAL cytokine, chemokine and cell infiltration) early during infection, "restoring" the immune response of obese mice to a "normal phenotype"; in fact, obesity delayed the immune response, rather than completely inhibiting it.

Exercise affects energy balance as well as leptin response, Type I IFN responsiveness, muscle PDC activation, furthermore increases serum anti-influenza virus-specific IgG2c antibody and $\mathrm{CD} 8+\mathrm{T}$ cell percentage in BAL; all mechanisms may be essential in host protection from infection [30, 32].

In summary, regular physical exercise emerges as a cornerstone as a preventive measure to improve host defense against influenza viral infection and other metabolic diseases in obese subjects (but not only). Furthermore, the increase in overweight and metabolic syndrome among adolescents in US $[33,34]$ and other industrialized countries $[35,36]$ constitutes an additional problem. In fact, being social distancing the only preventive measure that can be taken waiting for a vaccine against COVID-19, children and adolescents constitute a population where the actuation of social distancing is more difficult than in adults and where, apparently, there is a less symptomatic disease with a higher chance of having asymptomatic virus carriers.

\section{Is there an Immunomodulatory therapy to prevent influenza?}

The strongest, non-pharmacological, positive immunomodulatory intervention is physical exercise [37]. Moderate aerobic exercise (fit-walking, biking, swimming, jogging) has an anti-inflammatory effect decreasing alpha-TNF, MCP-1 and IL-6 and increasing IL-10 [37]. In addition, physical exercise causes an improvement in the hormone milieu increasing adiponectin and insulin sensitivity and decreasing insulin and leptin levels, reversing the altered hormone milieu of obese patients (Fig. 1). Along this line, Zheng et al. showed that regular exercise enhances the immune response against microbial antigens in humans [19]. It is interesting to note that positive immunomodulation can be achieved only with mild-to-moderate intensity exercise. In contrast, highintensity or prolonged exercise (like running a marathon) is known to deplete the immune response, mainly due to the increase in endogenous cortisol [37]. The basic cellular mechanism via which physical exercise exerts most of its effect is an activation of AMP-kinase [38].

Besides moderate physical exercise, two classes of drugs commonly used in obese and/or diabetic patients were shown to positively modulate the immune system.

1. AMPK activators. Metformin (MET) is a firstline drug for the treatment of type 2 diabetes mellitus and is used often off label to treat several forms of obesity-related disease (e.g., PCOS). Metformin is a potent stimulator of AMPK activation, and as we proved, it reduces sedentariness, increasing the activity in rodents [38]. This study demonstrated that metformin improves mice physical aerobic performance, ameliorates myotubes formation and prevents oxidative stress damage, modulating ERK and SOD (superoxide dismutases) signaling; therefore, we hypothesized that MET could also act as an antioxidant agent [37].

Other published results have suggested that MET has direct anti-inflammatory effects: For example, MET reduces lipopolysaccharide-induced pro-inflammatory responses in monocytes and macrophages, down-regulates Th17 cells, decreases TNF- $\alpha$ receptor signaling and NF-kB activation in endothelial cells and smooth muscle cells.

Diaz et al., in vivo and in vitro, observe a reduced B cellintrinsic inflammation and an improved antibody response with a consequent increase in the response to the influenza vaccine in type 2 diabetes patients taking MET. In conclusion, their results show that MET improves B cell responses through reduction in $\mathrm{B}$ cell-intrinsic inflammation and increased AMPK function, so AMPK may be a therapeutic target [39].

2. More specific AMPK (AMP-activated protein kinase) activators like AICAR (aminoimidazole carboxamide ribonucleotide) are still under study as immune modulators. AMPK is an enzyme that exerts anti-inflammatory effect upon activation, and it is also recognized as a stimulator of PPARs [40, 41]. In a murine model, Zhao et al. demonstrated that the administration of AICAR resulted in a decreased severity of lung injury and pro-inflammatory processes, as determined by diminished neutrophil accumulation and lowered levels of TNF- $\alpha$ and IL- 6 in bronchoalveolar lavage fluid [42].

Moseley et al. reported improved survival in influenza virus-infected mice treated with AICAR and a further improved survival (by 60\%) using combination therapy (AICAR + pioglitazone). The authors conclude that the results of their study provide preliminary evidence that 
stimulating the PPAR axis (with AICAR and PPAR $\gamma$ agonists) may prove to be a beneficial effect in the severe influenza infection [41].

3. PPAR gamma activators. Peroxisome proliferator-activated receptors (PPARs) are key regulators of inflammation and lipid metabolism, their activation results specifically in a reduction in inflammatory cytokines (as IL6, INF $\gamma$ ) [40]. Rosiglitazone and pioglitazone (two PPAR $\gamma$ agonists) are insulin-mimetic drugs used in type 2 diabetes with high overall potential in reducing influenza virus infection [40]. Pioglitazone seems to suppress inflammation by reducing MCP-1 expression, an important mediator of host inflammation [40]. Pioglitazone was shown to increase survival of infected mice up to $40 \%$, and the benefit may be attributed to modulation of subject inflammatory response [40, 41]. The combination of pioglitazone and AICAR increase survival of infected mice from 30 up to $100 \%$ [41]. Therefore, metformin, AICAR and PPAR $\gamma$ agonists, in combination or alone, might be studied and then used in the treatment of severe influenza in association with other antiviral/immunomodulatory drugs in the future.

\section{Conclusions}

Basal hormone milieu, defective response of both innate and adaptive immune system and sedentariness are major determinants in the severity of influenza viral infection in obese patients. Being obese not only increases the risk of infection and of complications for the single obese person, but recent evidence indicates that a large obese population increases the chance of appearance of more virulent viral strain, prolongs the virus shedding throughout the total population and eventually may increase overall mortality rate of an influenza pandemic. Waiting for the development of a vaccination against COVID-19, stated that isolation of positive cases and social distancing are the primary intervention, evidence from previous influenza pandemics suggests the following for our obese and obese-diabetic patients: 1 . lose weight with a mild caloric restriction. In addition to the obvious positive effect of weight loss, caloric restriction activates AMP-kinase, potentiating the immunomodulatory effect of physical exercise; 2 . include metformin and pioglitazone (when not contraindicated) in the drug treatment for type 2 diabetes; and 3. practice mild-to-moderate physical exercise in order to potentiate positive immunomodulation. Finally, present evidence suggests a prolongation of quarantine period in adult and adolescents obese patients.

Acknowledgements We thank Ms. K. Biasi for English language revision. This work was supported by Italian Ministry of Health-Ricerca Corrente-IRCCS Multimedica.

\section{Compliance with ethical standards}

Conflict of interest The authors declare that they have no conflict of interest.

Ethical standards This article does not contain any studies with human or animal subjects.

Informed consent For this type of article, formal consent does not apply.

\section{References}

1. Short KR, Kedziereska K, van de Sandt CE (2018) Back to the future: lessons learned from the 1918 influenza pandemic. Front Cell Infect Microbiol 8:1-19

2. Yang L, Chan KP, Lee RS et al (2013) Obesity and influenza associated mortality: evidence from an elderly cohort in Hong Kong. Prev Med 56(2):118-123

3. Moser J-AS, Galindo-Fraga A, Ortiz-Hernández AA et al (2019) Underweight, overweight, and obesity as independent risk factors for hospitalization in adults and children from influenza and other respiratory viruses. Influenza Other Respi Viruses 13:3-9

4. Morgan OW, Bramley A, Fowlkes A et al (2010) Morbid obesity as a risk factor for hospitalization and death due to 2009 pandemic influenza A (H1N1) disease. PLoS ONE 5:e9694

5. Caspard H, Jabbour S, Hammar N, Fenici P, Sheehan JJ, Kosiborod M (2018) Recent trends in the prevalence of type 2 diabetes and the association with abdominal obesity lead to growing health disparities in the USA: an analysis of the NHANES surveys from 1999 to 2014. Diabetes Obes Metab 20(3):667-671

6. Roser M, Ritchie H, Ortiz-Ospina E (2020) Coronavirus Disease (COVID-19). Research and Statistics. University of Oxford

7. Anderson CJ, Murphy KE, Fernandez ML (2016) Impact of obesity and metabolic syndrome on immunity. Adv Nutr 7:66-77

8. Ouchi N, Parker JL, Lugus JJ, Walsh K (2011) Adipokines in inflammation and metabolic disease. Nat Rev Immunol 11(2):85-97

9. Richard C, Wadowski M, Goruk S et al (2017) Individuals with obesity and type 2 diabetes have additional immune dysfunction compared with obese individuals who are metabolically healthy. BMJ Open Diabetes Res Care 5(1):e000379

10. Ahn SY, Sohn SH, Lee SY et al (2015) The effect of lipopolysaccharide-induced obesity and its chronic inflammation on influenza virus-related pathology. Environ Toxicol Pharmacol 40(3):924-930

11. Karlsson EA, Hertz T, Johnson C, Mehle A, Krammer F, SchultzCherry S (2016) Obesity Outweighs Protection Conferred by Adjuvanted Influenza Vaccination. mBio 7(4):1-12

12. Xue K, Stevens-Ayers T, Campbell AP et al (2017) Parallel evolution of influenza across multiple spatiotemporal scales. eLife 6:e26875

13. Zhang AJ, To KK, Li C et al (2013) Leptin mediates the pathogenesisi of severe 2009 pandemic influenza A (H1N1) infection associated with cytokine dysregulation in mice with diet-induced obesity. J Infect Dis 207:1270-1280

14. Park S, Jeon J-H, Min B-K et al (2018) Role of the pyruvate dehydrogenase complex in metabolic remodeling: differential pyruvate dehydrogenase complex functions in metabolism. Diabetes Metab J. 42:270-281

15. Honce R, Schultz-Cherry S (2019) Impact of Obesity on Influenza, a virus Pathogenesis, Immune Response, and Evolution. Front Immunol 10:1-15 
16. O’Brien KB, Vogel P, Duan S et al (2012) Impaired wound healing predisposes obese mice to severe influenza virus infection. $\mathrm{J}$ Infect Dis 205:252-261

17. Klinkhammer J, Schnepf D, Ye L et al (2018) INF-lambda prevents influenza virus spread from the upper airways to the lungs and limits virus transmission. elife 7:e33354

18. Honce R, Karlsson EA, Wohlgemuth N et al (2020) Obesityrelated microenvironment promotes emergence of virulent influenza virus strains. mBio 11(2):1-16

19. Zheng Q, Cui G, Chen J et al (2015) Regular exercise enhances the immune response against microbial antigens through upregulation of toll-like receptor signaling pathways. Cell Physiol Biochem 37:735-746

20. Reidy PT, Yonemura NM, Madsen JH et al (2019) An accumulation of muscle macrophages is accompanied by altered insulin sensitivity after reduced activity and recovery. Acta Physiol 226:1-16

21. Wong GCL, Narang V, Yanxia L et al (2019) Hallmarks of improved immunological responses in the vaccination of more physically active elderly females. Exer Immunol Rev 25:20-33

22. Maier H, Lopez R, Sanchez N et al (2018) Obesity increased the duration of influenza A virus shedding in adults. J Infect Dis 218(9):1372-1382

23. Yan J, Grantham M, Pantelic J et al (2018) Incetious virus in exhaled breath of syntomatic seasonal influenza cases from a college community. PNAS 115(5):1081-1086

24. Atkinson RL, Dhurandhar NV, Allison DB et al (2005) Human adenovirus-36 is associated with increased body weight and paradoxical reduction of serum lipids. Int J Obes 29:281-286

25. Na H-N, Nam J-H (2012) Adenovirus 36 as an obesity agent maintains the obesity state by increasing MCP-1 and inducing inflammation. JID 205:914-922

26. Vangipuram SD, Yu M, Tian J et al (2007) Adipogenic human adenovirus-36 reduces leptin expression and secretion and increases glucose uptake by fat cells. Int J Obes 31:87-96

27. Pasarica M, Shin AC, Dhurandhar NV (2006) Human adenovirus 36 induces adiposity, increases insulin sensitivity, and alters hypothalamic monoamines in rats. Obesity 14:1905-1913

28. Vangipuram SD, Sheele J, Atkinson RL et al (2004) A human adenovirus enhances preadipocyte differentiation. Obes Res 12:770-777

29. Ritter JB, Wahl AS, Freund S, Genzel Y, Reichl U (2010) Metabolic effects of influenza virus infection in cultured animal cells: intra- and extracellular metabolite profiling. BMC Syst Biol 13:4-61

30. Dumitru CT (2013) Regulation of muscle pyruvate dehydrogenase complex in insulin resistance: effects of exercise and dichloroacetate. Diabetes Metab J 37:301-314
31. Yamane K, Indalao IL, Chida J, Yamamoto Y, Hanawa M, Kido H (2014) Diisopropylamine dichloroacetate, a novel pyruvate dehydrogenase kinase 4 inhibitor, as a potential therapeutic agent for metabolic disorders and multiorgan failure in severe influenza. PLoS ONE 9(5):1-13

32. Warren KJ, Olson MM, Thompson NJ (2015) Exercise improves host response to influenza viral infection in obese and non-obese mice through different mechanisms. PLoS ONE 25:1-27

33. Lee AM, Ferin CR, Filipp SL, Gurka MJ, DeBoer MD (2017) Examining trends in prediabetes and its relationship with the metabolic syndrome in U.S. adolescents. Acta Diabetol 54(4):373-381

34. Hagman E, Besor O, Hershkop K et al (2019) Relation of the degree of obesity in childhood to adipose tissue insulin resistance. Acta Diabetol 56:219-226

35. Spreghini N, Cianfarani S, Spreghini MR et al (2019) Oral glucose effectiveness and metabolic risk in obese children and adolescents. Acta Diabetol 56:955-962

36. Milano Cities Changing Diabetes Atlas (2020) Diabete Tipo 2 e Obesità nell'area di Milano Città Metropolitana

37. Codella R, Luzi L, Inverardi L, Ricordi C (2015) The anti-inflammatory effects of exercise in the syndromic thread of diabetes and autoimmunity. Eur Rev Med Pharmacol Sci. 19:3709-3722

38. Senesi P, Montesano A, Luzi L, Codella R, Benedini S, Terruzzi I (2016) Metformin treatment prevents sedentariness related damages in mice. J Diabetes Res 2016:8274689. https://doi. org/10.1155/2016/8274689

39. Diaz A, Romero M, Vazquez T et al (2017) Metformin improves in vivo and in vitro $\mathrm{B}$ cell function in individuals with obesity and Type-2 Diabetes. Vaccine 35(20):2694-2700

40. Darwish I, Mubareka S, Conrad LW (2011) Immunomodulatory therapy for severe influenza. Expert Rev Anti Infect Ther 9(7):807-822

41. Moseley CE, Webster RG, Aldridge JR (2010) Peroxisome proliferator-activated receptor and AMP-activated protein kinase agonists protect against lethal influenza virus challenge in mice. Influenza Other Resp Virus 4:307-311

42. Zhao X, Zmijewski JW, Lorne E et al (2008) Activation of AMPK attenuates neutrophil proinflammatory activity and decreases the severity of acute lung injury. Am J Physiol Lung Cell Mol Physiol 295(3):L497-504

Publisher's Note Springer Nature remains neutral with regard to jurisdictional claims in published maps and institutional affiliations. 